


\title{
The roles of hydration and evaporation during the drying of a cement paste by localized NMR
}

\author{
Maxime Van Landeghem ${ }^{\text {a,b,c }}$, Jean-Baptiste d'Espinose de Lacaillerie a , Bernhard Blümich ${ }^{\text {b }}$, \\ Jean-Pierre Korb ${ }^{\mathrm{d}}$, Bruno Bresson ${ }^{\mathrm{a}, *}$ \\ a Sciences et Ingénierie de la Matière Molle, UMR 7615 CNRS UPMC, ESPCI ParisTech, 10 rue Vauquelin, 75005 Paris, France \\ b ITMC, RWTH-Aachen Worringer Weg 1, D52056 Aachen, Germany \\ c Saint-Gobain Recherche, 39 quai Lucien Lefranc, BP 135, 93303 Aubervilliers cedex, France \\ d Physique de la Matière Condensée, Ecole Polytechnique, CNRS, 91128 Palaiseau, France
}

\section{A R T I C L E I N F O}

\section{Article history:}

Received 25 July 2012

Accepted 29 January 2013

\section{Keywords:}

Drying (A)

Pore Size Distribution (B)

Transport Properties (C)

Humidity (A)

Hydration $(\mathrm{A})$

\begin{abstract}
A B S T R A C T
The moisture distribution during the setting of a thin mortar layer can be particularly complex to manage under dry $(20 \% \mathrm{RH})$ and hot weather (above $25{ }^{\circ} \mathrm{C}$ ) conditions. To better understand the fundamental phenomena at stake, we used static gradient relaxation NMR tools such as Profile MOUSE and STRAFI. This allowed disentangling the mutual effect of evaporation and self-desiccation by hydration. The interest of combining the two techniques is that the capillary water is observed with the MOUSE while STRAFI reveals quantitatively the build-up of the hydrate gel nanostructure. Spatially resolved and 2D relaxation exchange experiments on a model white cement paste revealed that although evaporation induced a capillary water gradient, the kinetics of the building of the pore structure and its homogeneity remained unaffected.
\end{abstract}

(C) 2013 Published by Elsevier Ltd.

\section{Introduction}

The knowledge of the moisture distribution in building materials and more generally the transport properties of the adsorbed water phase are a key-point for the understanding of their durability. Drying can induce cracking and is a matter of permanent concern for the mortar and concrete industry. At a smaller scale, the mechanical resistance of cementitious materials is linked to the density of the $\mathrm{C}-\mathrm{S}-\mathrm{H}$ phase which is largely determined by the relative volume of the water saturated $\mathrm{C}-\mathrm{S}-\mathrm{H}$ gel nanopores. The physics of drying has been described with great success both at the theoretical [1] and experimental [2] levels for model porous systems. However a cement paste represents a more complex challenge as the pore volume cannot be considered constant and water is a chemically reactive phase.

When a Portland cement powder is mixed with water, a chemical hydration reaction occurs [3]. The soluble components present in the cement powder, when in contact with water, dissolve quickly, creating a saturated solution from which the hydrates precipitate. The hydrates grow on the surface of the cement grain, connecting the grains to each other and hardening the then solid material. Cement-paste hardening is thus intrinsically accompanied by a liquid water consumption called self-desiccation. Self-desiccation corresponds to the transformation of part of the liquid water into solid hydrates. It must be distinguished from true drying which corresponds to the removal of liquid water by transfer to the gas phase, that is evaporation at the paste/air interface. The matter is further complicated by the possible interplay between self-desiccation and evaporation: the tortuous hydrate network affects the water transport toward the interface for evaporation, while one can expect, in turn, that evaporation, by decreasing the local water/cement ratio, affects the formation of the hydrate network. In the following, we will refer to the simultaneous effects of evaporation and self-desiccation as "drying".

The ultimate aim of our study is to assess during drying the mutual roles of evaporation and self-desiccation on the final porous structure. In that respect, the state and the distribution of water molecules within the cement paste is fundamental for the understanding of its evolving pore structure, and Nuclear Magnetic Resonance (NMR) is one of the few non invasive methods that can deliver this information. Indeed, it is now well established that proton NMR relaxation-rate measurements (both spin-spin relaxation referred to as $T_{2}$ relaxation and spin-lattice relaxation referred to as $T_{1}$ relaxation) give access not only to the water content but also to the saturated pore-size distribution of a hydrating cement paste.

For all kinds of cement, it is now accepted that the paramagnetic impurities at the surface of the pores control the NMR relaxation rate of all the water protons in the pore, even for white cement (with low concentration of $\mathrm{Fe}$ ). The relaxation process is the following: the 
water molecules undergo a 3D diffusion in the pore, and, at some point, a water molecule reaches the surface of the pore where its diffusion is restricted, before returning to the bulk of the pore [4]. During its stay on the surface, the water molecule can enter the vicinity of a paramagnetic impurity embedded within the pore wall. The magnetic interaction with the paramagnetic centers is then the dominant contribution to the relaxation, even for cement with low Fe content.

Since the surface residence time is very short compared to the NMR measurement time (fast exchange limit), the effective relaxation rate $\left(T_{2}^{\text {eff }}\right)^{-1}$ of water is an average of the bulk liquid relaxation rate $\left(T_{2}^{\text {bulk }}\right)^{-1}$ and the enhanced surface relaxation rate, weighted according to the surface-to-volume ratio $S / V$ of the pore [5]:

$\frac{1}{T_{2}^{\mathrm{eff}}}=\frac{1}{T_{2}^{\text {bulk }}}+\rho \frac{S}{V}$

where $\rho$ is the surface relaxivity. Thus, the relaxation rate reflects the pore size and morphology.

For the complete pore network, the total experimental magnetic decay $S(t)$ is the superposition of the exponential decays $T_{2}^{\text {effi }}$ characteristic of each pore size weighted by their relative population $A_{i}$ :

$S(t)=\sum_{n} A_{i} \exp \left(-\frac{t}{T_{2}^{\mathrm{eff} i}}\right)$.

Therefore, in principle, NMR relaxation analysis can provide the relative abundance of each water population in the cement paste (in the hydrate structure, in the C-S-H gel pores, and in the capillary pores) and can even provide the corresponding pore size distribution [6]. The problem is thus to recover the pore size distribution from the time relaxation of the NMR signal. The corresponding mathematical procedure is formally a Laplace inversion. This operation, a keypoint of the analysis, was recently facilitated by the availability of stable multi-dimensional algorithms executable on desktop computers [7].

In a typical white cement paste hydrated during a few days with a water/solid ratio of 0.4 , the extraction of each $T_{2}^{\text {effi }}$ component of the relaxation leads to a discrete distribution which ranges from $10 \mu \mathrm{s}$ up to $1 \mathrm{~s}$ [8]. Numerous NMR experiments carried out at different frequencies (from $\mathrm{kHz}$ up to $\mathrm{MHz}$ ) have led to the following conclusions: all the water molecules in a hydrated cement paste can be categorized as belonging to different relaxation components. The first component is fast relaxing with $T_{2}^{\text {eff }} \mathrm{S}$ of $15 \mu \mathrm{s}$ [9]. It corresponds to the hydroxyl group in the Portlandite. The second component with $T_{2}^{\text {eff }}$ between $100 \mu \mathrm{s}$ and $400 \mu \mathrm{s}$ corresponds to the water associated to the hydrate nanostructure. In this category, the structural water of the $\mathrm{C}-\mathrm{S}-\mathrm{H}$ interlayer and the water contained in the nanopores of the $\mathrm{C}-\mathrm{S}-\mathrm{H}$ gel were included. Indeed, the $\mathrm{C}-\mathrm{S}-\mathrm{H}$ is inherently nanoporous, and although the water contained in those very small pores $(2 \mathrm{~nm})$ is not chemically speaking structural, its removal would imply the development of extreme capillary pressures that would destroy the gel nanostructure as well. Another $T_{2}^{\text {eff }}$ component of the cement paste proton NMR signal ranges from about $1 \mathrm{~ms}$ to $200 \mathrm{~ms}$. It arises from water in pores scaling from a few nanometers up to less than a micrometer and is called the capillary water. The specificity of this type of water is that it can be reversibly removed without any destruction of the nanostructure of the cement paste. Finally, a last component of slowly relaxing protons (circa $1 \mathrm{~s}$ ) corresponds to free liquid-like water. It must be noted upfront that in presence of a gradient, this population cannot be distinguished as its $T_{2}^{\text {eff }}$ is compressed by diffusive effects toward the range of $T_{2}$ 's capillary water. In this study, we placed a cut-off at $T_{2}^{\text {eff }}$ equal to $150 \mu \mathrm{s}$ which means that all the water moistures with a $T_{2}^{\text {eff }}$ below $150 \mu$ s are included in the same component (hydroxyl groups and gel water). Table 1 summarizes the $T_{2}^{\text {eff }}$ values that were actually accessible in our study.
$T_{2}^{\text {eff }}$ values actually accessible versus pore size and proton populations

\begin{tabular}{lll}
\hline$T_{2}^{\text {eff }}$ range & Pore size according to [8] & Proton population \\
\hline Above $150 \mu \mathrm{s}$ & $2 \mathrm{~nm}$ & "Gel" water \\
$1 \mathrm{~ms}$ to $200 \mathrm{~ms}$ & $\begin{array}{l}(1 \mathrm{~ms}) 16 \mathrm{~nm} \\
(50 \mathrm{~ms}) 170 \mathrm{~nm}\end{array}$ & Capillary water \\
& $(200 \mathrm{~ms}) 270 \mathrm{~nm}$ & \\
$1 \mathrm{~s}$ & & Free water
\end{tabular}

The same conclusions can be essentially drawn from $T_{1}$ relaxation rates analysis. By $T_{2}$ analysis even without a predictive relaxation model, some authors [10] were able to draw precise conclusions about the evolution of each kind of water during the hydration cement paste.

To follow the drying, the simplest study can be performed on the bulk material. However, to actually discriminate drying and desiccation mechanisms, the monitoring of the total water in the sample versus time is not sufficient. Due to the anisotropy of the evaporation process, it is necessary to follow the distribution and evolution of water within the pore system not only in time but also in space. This justifies the combination of NMR relaxation with MRI (Magnetic Resonance Imaging) techniques. As all of the evaporation occurs at the paste/air interface, one-dimensional profiling methods are the most adequate tool. When dealing with a cement paste, the MRI technique must rely on stable and large gradient to overcome the rapid relaxation rates (see Table 1 ). This reduces the techniques of choice to the ones involving strong static gradients, such as STRAY Field Imaging (STRAFI). Indeed, there is already a significant body of literature concerning the study cement paste using the high stray fields of superconducting magnets [11-15], but never as a function of the temperature and humidity, which however influences deeply the drying. As a result, most studies have focused on self-desiccation and pore network built-up. Alternatively, some authors have chosen to use single sided permanent magnets with lower fields and static gradients such as the GarField [16] or the Profile NMR MOUSE [17].

The present study is thus by far not the first one to apply STRAFI or single-sided magnet methods to the setting of a cement paste but here we focus on the simultaneous effects of self-desiccation and evaporation by varying the temperature and humidity conditions. Furthermore, we combined the two complementary NMR profiling methods: NMR imaging in the Stray Field of the superconducting magnet (referred to as STRAFI experiment in the following) $[14,18]$ and imaging by NMR-MObile Universal Surface Explorer (referred to as Profile MOUSE experiment in the following) [17]. The versatile Profile MOUSE experiment is a powerful tool to profile the water distribution in a cement paste at different temperature as it is portable and can easily fit into climate chambers. In a first part, the Profile MOUSE results are presented. They show the effect of drying on the upper face of the paste (the face in contact with air) and the effect of temperature on the water distribution as a function of the depth in the sample. The complete interpretation of the drying process needs the observation of all kinds of water in the cement (free water or water in the pore structure, gel water, as well as structural water in the hydrates). The STRAFI experiments carried out with a home-made NMR probe combine imaging (open geometry of the probe so that the sample can be moved through the coil) and solid state NMR advantages (short pulse, short dead time, short echo time). In a second part of the paper, the evolution of the porous network in a slice of the paste is presented. It is shown how bulk proton NMR relaxation experiments usually performed in a cement paste ( $T_{1}-T_{2}$ correlation maps, $T_{1}$ and $T_{2}$ distribution) can be adapted to be spatially resolved. In a third part of the paper, the effect of relative humidity is studied independently of temperature. In the last part, the combined effect of temperature and relative humidity is addressed. 


\section{Materials and methods}

The principle of the Profile MOUSE [19,17] and STRAFI [14,18] experiments has been described elsewhere and only the main features of the experiment are recalled.

\subsection{Profile MOUSE setup for studies with humidity and temperature control}

The Profile MOUSE used in this work is a unilateral NMR sensor which is specially designed to generate a magnetic field with a uniform gradient in a sensitive volume located at $25 \mathrm{~mm}$ over the surface of the magnet [19,17]. This allows 1D profiling over a range of $25 \mathrm{~mm}$ at a magnetic field of $0.32 \mathrm{~T}\left({ }^{1} \mathrm{H}\right.$ frequency of $13.8 \mathrm{MHz}$ ). The magnetic field gradient over the sensitive slice is $7.5 \mathrm{~T} / \mathrm{m}$. The magnet is mounted on a lift which allows its movement in relation to the sample. Due to the presence of a magnetic field gradient, when a finite radiofrequency pulse is emitted at the frequency of $13.8 \mathrm{MHz}$, only a thin slice (typically $100 \mu \mathrm{m}$ ) is excited. This thin slice is excited using a CPMG sequence in order to measure its apparent transverse relaxation rate $T_{2}^{\text {app }-1}[20]$. The apparent $T_{2}^{\text {app }}$ for a species of diffusion coefficient $D$ is related in presence of a gradient $G$ to the $T_{2}^{\text {eff }}$ through the following formula:

$\frac{1}{T_{2}^{\mathrm{app}}}=\frac{1}{T_{2}^{\mathrm{eff}}}+D \cdot \frac{\gamma^{2} G^{2} t_{\mathrm{E}}^{2}}{12}$

where $\gamma$ is the gyromagnetic ratio and $t_{\mathrm{E}}$ is echo time of the CPMG sequence. Hereafter, it must be understood that what is referred as $T_{2}$ is actually $T_{2}^{\text {app }}$ in order to keep a compact notation.

The echo time was set to $140 \mu$ s. Once the signal arising from this slice is acquired, the lift moves the magnet in order to measure the next step of the profile. The Profile MOUSE mounted on its lift fits a $38 \times 38 \times 38 \mathrm{~cm}^{3}$ box and can easily be introduced in a commercial temperature and humidity cabinet (see Fig. 1). This cabinet (model LHU-113) was provided by ESPEC $\circledast$.

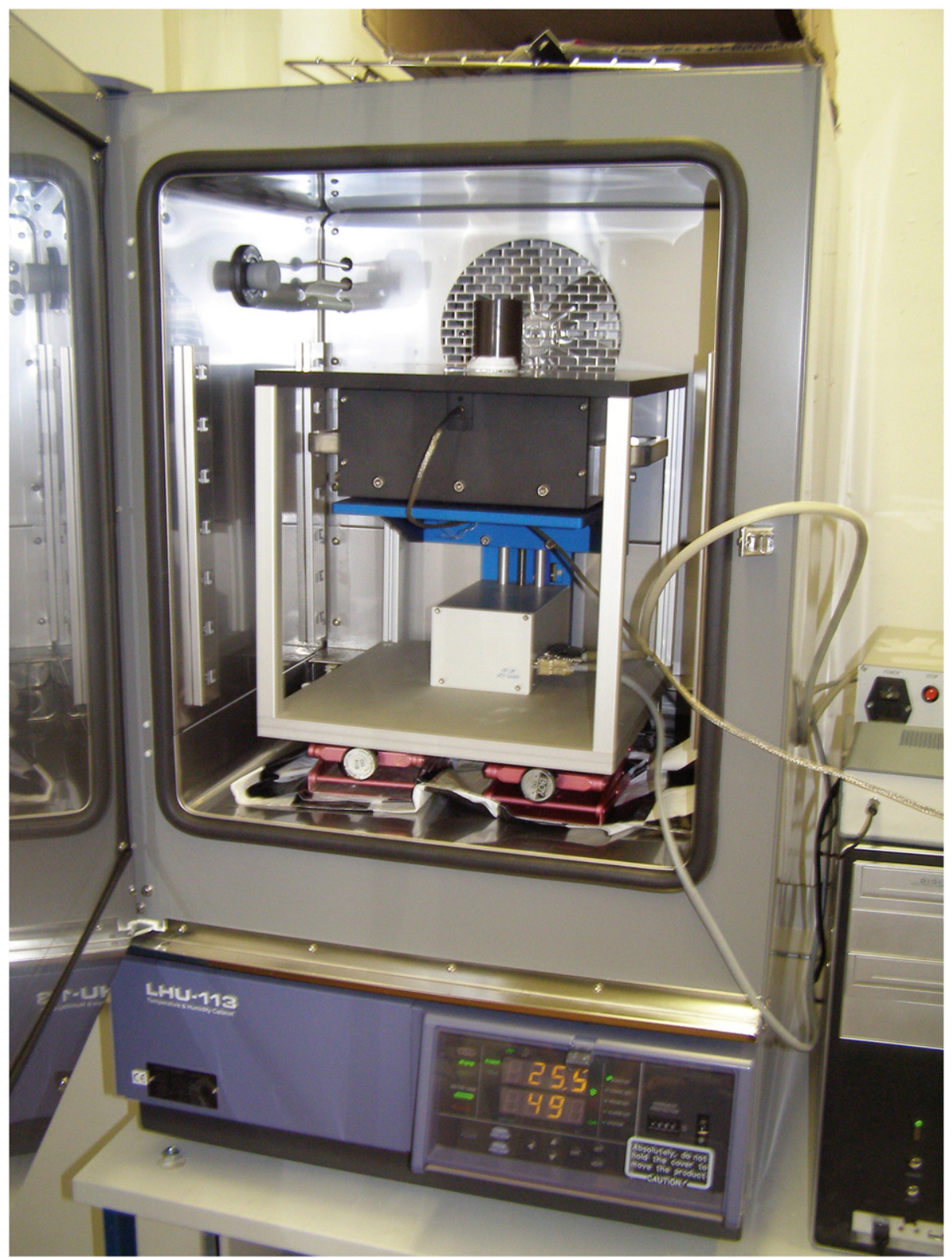

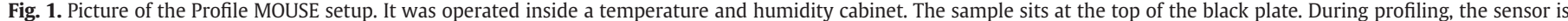
moved step by step from top to bottom relative to this plate so the whole profile can be scanned. 


\subsection{STRAFI setup}

STRAFI can be seen as a high field version of the Profile MOUSE. In the STRAFI, the sample is placed out of the bore of the superconducting NMR magnet, along the magnet axis. It is then moved to a location where the static magnetic field fringe gradient from the superconducting magnet is homogeneous across a slice perpendicular to the axis of the magnet. As with the profile MOUSE, this slice is used for imaging. We recently reported [18] how to find this position in the fringe field and the way to align the sample with respect to the superconducting magnet so that a spatial resolution of $5 \mu \mathrm{m}$ could be achieved on a sample with a lateral extension of $1 \mathrm{~cm}$. From then on, the procedure to complete the profile of the sample is comparable to the one described for the Profile MOUSE, except that the sample is mobile and not the magnet. The sample is placed on top of a rod driven by a lift which repositions it between each NMR acquisition. Besides the high resolution which can be achieved with this setup, STRAFI exhibits another significant advantage arising from the high static gradient and the use of a solid-like imaging probe at high field (homemade): it allows access to very short $T_{2}$ values (typically hundreds of microsecond using CPMG detection train with echo times of the order of a few tens of $\mu$ ). Indeed, the ring-down time decreases with increasing frequency. This means that it is possible to combine good spatial resolution, measurement of short $T_{2}$ values such as that of gel water in $\mathrm{C}-\mathrm{S}-\mathrm{H}$ and benefit from the high field detection. In fact, the magnetic field at which our STRAFI experiments were carried out on our Oxford $7 \mathrm{~T}$ magnet was $3.2 \mathrm{~T}$, corresponding to a proton larmor frequency of $137 \mathrm{MHz}$. At this position, the value of the magnetic field gradient was about $52 \mathrm{~T} / \mathrm{m}$. This high static gradient (as well as the one of the Profile MOUSE) is the key to success for imaging rigid materials. Indeed, it is sufficiently large to overcome any loss of resolution arising in solids from magnetic susceptibility gradients and dipolar interactions [21].

The STRAFI probe was fitted by a humidity and temperature control custom-made setup (Fig. 2). The cement paste was poured in $30 \mathrm{~mm}$ tube. The thickness of the cement paste was $10 \mathrm{~mm}$ and the upper surface was in contact with air. The temperature was controlled by a commercial NMR temperature unit manufactured by Eurotherm ${ }^{\circledR}$. A thermocouple placed close to the sample allowed the regulation of a heater inside a dewar positioned at the bottom of the probe. The temperature which could be maintained ranged between $20^{\circ} \mathrm{C}$ (ambient temperature) and $80^{\circ} \mathrm{C}$. The humidity was regulated by manually mixing dry air with water saturated air drawn from a bubbler. This setup allowed humidity levels from 10 to $70 \% \mathrm{RH}$.

\subsection{Preparation of the white cement paste}

The white cement was provided by Saint-Gobain Recherche (white cement CEM II) and used as received. The cement paste was prepared with a water/cement ratio of 0.4. In the case of experiments using the Profile MOUSE, the paste was prepared in a conventional $250 \mathrm{~mL}$ container, directly placed on the plate above the sensor. For the STRAFI measurements, the water and cement pastes were cast into a $10 \mathrm{~mm}$ glass tube. The unsealed tube was then introduced into the glass cylinder of our homemade probe which is used to guide the sample and regulate humidity and temperature.

\section{Results and discussion}

\subsection{Complementarity of the profile MOUSE and STRAFI}

The determination of the pore structure of cement by proton NMR requires the measurement of the signal decay (see Eq. (2)). This measurement was performed using the CPMG sequence which consists of a $90^{\circ}$ pulse followed by a train of $180^{\circ}$ pulses $[22,23]$. Each echo was recorded independently. The first echo obtained was removed as its amplitude is determined by factors different from those of the other echoes [23]. The obtained decay was then either fitted by multiexponential functions or inverted using the inverse Laplace transformation (ILT) algorithm. Besides the fine dependence of $T_{2}$ with the pore size (see Table 1 ), two $T_{2} \mathrm{~S}$ (or distribution of $T_{2} \mathrm{~S}$ ) are usually expected in white cement paste in presence of a gradient. As stated in the introduction, this corresponds to water in capillary pores and structural water in the hydrates. Fig. 3 exhibits typical CPMG decays of a white cement paste obtained using the Profile MOUSE (a) and STRAFI (b). Both decays corresponded to a drying of $8 \mathrm{~h}$. The associated ILT is exhibited in Fig. 3 ( $c$ and d), respectively. In the case of the Profile MOUSE, the distribution of $T_{2} \mathrm{~S}$ seemed mono-modal. The single peak was attributed to capillary water. The echo time of the Profile MOUSE $(140 \mu \mathrm{s})$ does not allow to see relaxing species with a $T_{2}$ shorter than a few hundreds of microseconds. Thus, "gel" water and structural water are naturally filtered out when white cement is measured with this tool. On the contrary, the $T_{2}$ distribution recorded by STRAFI was bimodal (see Fig. 3d). This could be achieved because the echo time in the CPMG detection is much shorter (40 $\mu \mathrm{s})$. The two peaks are attributed to capillary water ( $T_{2}$ around $1 \mathrm{~ms}$ ) and "gel" water ( $T_{2}$ around $100 \mu$ s). Still the chemically structural water ( $T_{2}$ expected around $10 \mu \mathrm{s}$ ) cannot be seen by STRAFI. As a consequence, this preliminary study shows that the two tools complement each other. The Profile MOUSE being sensitive to long $T_{2} \mathrm{~S}$ is a simple indicator for change in capillary water content within white cement due to transfer to the interface such as during evaporation. The STRAFI being sensitive also to "gel" water is expected to provide more information about the pore structure.

\subsection{Evolution of the pore structure of white cement}

\subsection{1. $1 D$ experiment}

To follow the evolution of the porous structure of white cement, a sample of white cement paste was prepared and measured by STRAFI using a CPMG detection train. This measurement was performed at a depth of $10 \mathrm{~mm}$ from the surface of the sample in order to accumulate more scans and obtain the better signal-to-noise ratio necessary for a good ILT. The experiment lasted one day, and one decay was measured every $17 \mathrm{~min}$. Afterwards, each decay was numerically inverted to provide the $T_{2}$ distribution versus the hydration time (Fig. 4a) while a double exponential fit was also performed. The amplitudes of the two decays are displayed in Fig. 4b. During the first $4 \mathrm{~h}$ of hydration, a single $T_{2}$ could be realistically defined with a good level of confidence and the distribution could be considered to be mono-modal, going from a $T_{2}$ of $4.5 \mathrm{~ms}$ at time $t=0$, to $2 \mathrm{~ms}$ at $t=$ $5 \mathrm{~h}$. Then a shorter $T_{2}$ population appeared after a hydration time of $10 \mathrm{~h}$, while the amplitude of the population with the long $T_{2}$ decreased below the amplitude of the population with a short $T_{2}$. This corresponded to the setting time of the paste and the formation of the percolating $\mathrm{C}-\mathrm{S}-\mathrm{H}$ gel network. The long $T_{2}$ value shortened progressively from $1 \mathrm{~ms}$ down to $400 \mu$ s between $10 \mathrm{~h}$ and $20 \mathrm{~h}$ while the shorter $T_{2}$ was constant at about $150 \mu$ s.

Previous work [8] helped us to clearly attribute each kind of water to details of the pore structure. For example, $T_{2}=5 \mathrm{~ms}$ observed at a drying time of about $6 \mathrm{~h}$ can be attributed to capillary pores of radius $r=100 \mathrm{~nm}$, and $T_{2}=1 \mathrm{~ms}$ has previously been attributed to pores of radius $r=15 \mathrm{~nm}$. The short $T_{2}$ of $150 \mu$ s corresponds to the gel $\mathrm{C}-\mathrm{S}-\mathrm{H}$ nanopores of a few nanometers in diameter. However, the $T_{2}$ of the chemically bound water $(10-30 \mu \mathrm{s})$ [12] (C-H and chemically structural water of $\mathrm{C}-\mathrm{S}-\mathrm{H}$ ) was not observed in the previous analysis. Actually, it must be recalled that the first echo of the CPMG train was removed as it is known to be weighted by a factor $2 / 3$ in the presence of a magnetic field gradient $[20,23]$. However, we noticed that the first echo occurring $30 \mu$ after the first pulse was much more intense than the others. This could be interpreted as the contribution of the 


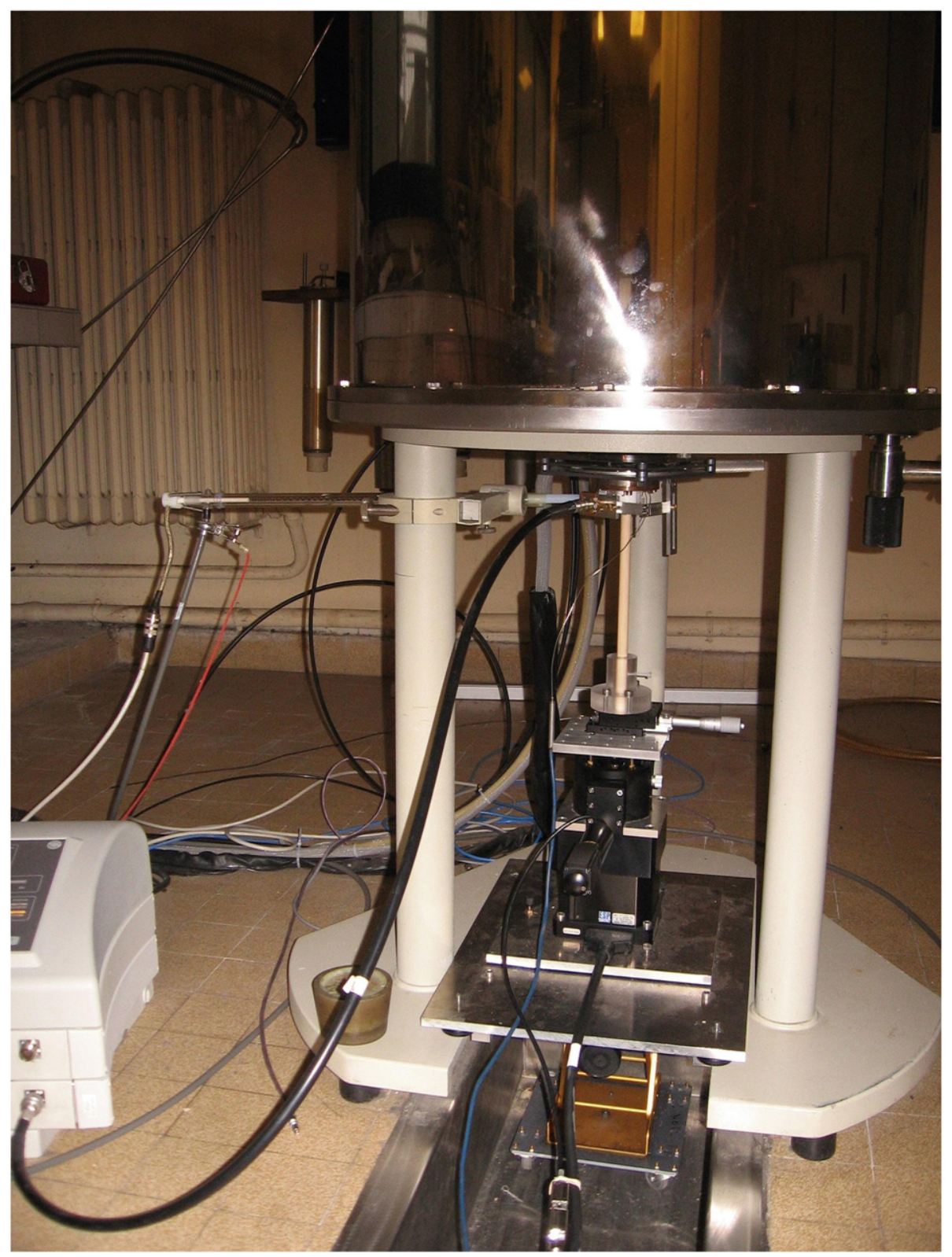

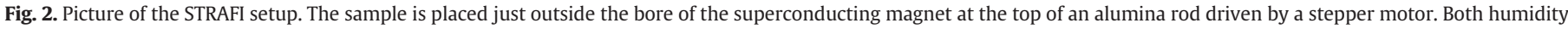
and temperature are regulated.

chemically bound water in cement hydrates which would have not completely relaxed yet. In order to stabilize the analysis, we nevertheless stuck to our choice of removing the first point before processing the data on Fig. 4 as well as in the following analysis.

The advantage of the experiment presented here is the clear attribution of the different kinds of water which successively appear in the material. It also evidences the discrete characteristic of the pore network developing with the time. The interesting result is that it can be performed with spatial resolution. An example of this kind of analysis is depicted in Fig. 5. Fig. 5a exhibits profiles of white cement measured at various hydration times using only the first echo of the CPMG echo train. At $25{ }^{\circ} \mathrm{C}$ and $30 \% \mathrm{RH}$ a clear variation of the water distribution is observed along the sample. To check if drying affects or not the kinetics of structuring of the material, a $T_{2}$ analysis similar to the one of Fig. 4b was performed at various heights in the sample: at the surface of the sample, at a depth of $3 \mathrm{~mm}$ from the surface and at a depth of $10 \mathrm{~mm}$. The kinetics of emergence of the population with the short $T_{2}$ (red curve) as well as that of the decrease of the population with long $T_{2}$ at these positions (Fig. 5b, c, and d) were virtually identical at this position. This showed that the kinetics of the structuring was not affected by the drying.

\subsection{2. $2 \mathrm{D}$ experiment}

The previous 1D studies performed by STRAFI at a single height can be completed by 2D $T_{1}-T_{2}$ NMR experiments which can be adapted for use in inhomogeneous magnetic field [24], and some applications to the study of cement pastes have been reported $[16,25]$. The sequence we used was a saturation recovery sequence [26] followed by a mixing time $\tau$ varying logarithmically [22] and a CPMG detection train.

A $T_{1}-T_{2}$ 2D NMR experiment was carried at a fixed position, in the middle of the paste. The $2 \mathrm{D}$ data were processed by $2 \mathrm{D}$ inverse Laplace transformation adapted to the study of $T_{1}-T_{2}$ correlations [27]. Fig. 6 displays the $T_{1}-T_{2}$ maps of a white cement paste $(\mathrm{w} / \mathrm{c}=0.4)$ for two hydration times. After $6 \mathrm{~h}$ of hydration (Fig. $6 \mathrm{a}$ ), the 2D correlation map of the white cement exhibited a single peak at $\left(T_{1}=15 \mathrm{~ms}\right.$ and 

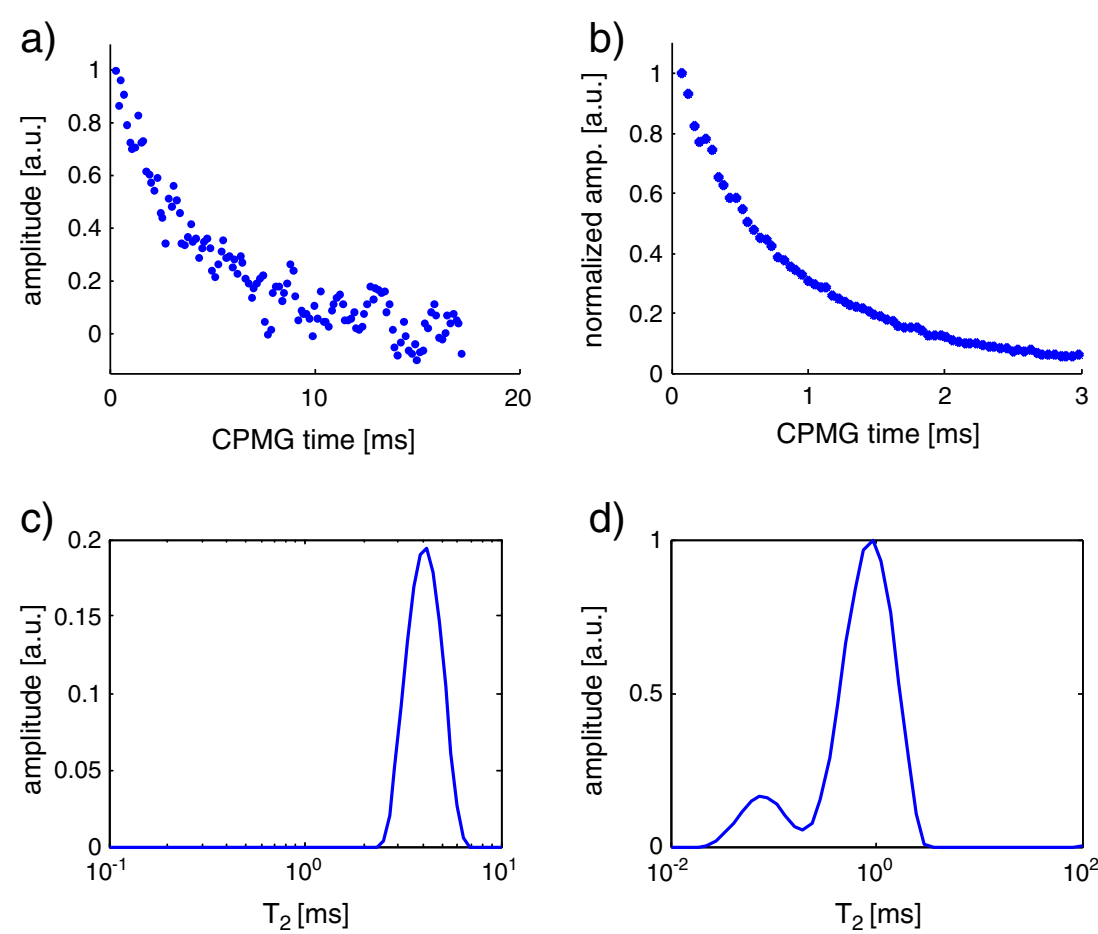

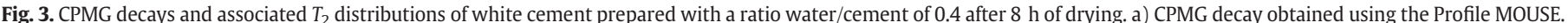

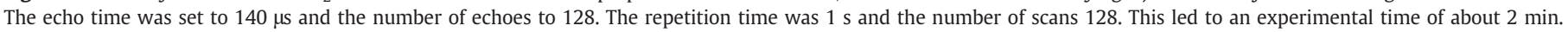

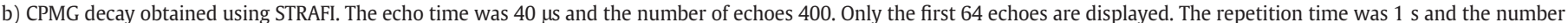

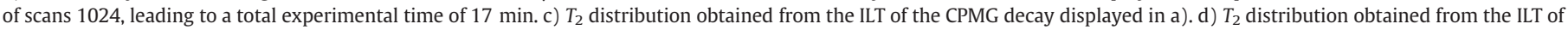
the CPMG decay displayed in b).

$T_{2}=4 \mathrm{~ms}$ ). Thus, at this time, the pore network appeared to be a completely percolated continuous phase. At time 4 days (Fig. 6b), a peak around $T_{1}=2-3 \mathrm{~ms}$ and $T_{2}=400 \mu \mathrm{s}$ appeared along with a peak at $T_{1}=800 \mu \mathrm{s}$ and $T_{2}=150 \mu \mathrm{s}$. The first noticeable result was that the peaks stay on the line $T_{1}=5 T_{2}$, demonstrating that the behavior of the water molecules followed the theory briefly sketched in the introduction [8]. Indeed, one of the consequences of this theory is the dependence of the ratio $T_{1} / T_{2}$ on a few set of predictable parameters (namely the surface hoping time and the residence time on the surface of the pore). The second remark is that on the time scale of the experiment, no exchange peak was observed for 4-day hydration. Thus the two porous networks observed at that stage were almost disconnected. The third remark is that according to the literature [8], the difference between the correlation map shown in Fig. $6 a$ and $b$ could be interpreted in the following way: the disappearance of the peak at $T_{1}=15 \mathrm{~ms}$ and $T_{2}=2.5 \mathrm{~ms}$ was due to the conversion of the capillary water in large pores into capillary water in smaller pores of $T_{1}=2 \mathrm{~ms}$ and $T_{2}=400 \mu \mathrm{s}$ and into C-S-H "gel" water of $T_{1}=800 \mu$ s and $T_{2}=150 \mu$ s. The $\mathrm{C}-\mathrm{S}-\mathrm{H}$ gel had then significantly evolved. This interpretation supports the discrete interpretation of the pore structure.

\subsubsection{Conclusion of the STRAFI experiments}

The experiments complement each other and global conclusions can be drawn. First of all, from Figs. 4a and 6b, it appeared that the relaxation time $T_{2}=150 \mu \mathrm{s}$ was invariant. The exchange with the network was weak. Indeed, if we attribute this $T_{2}$ to the $2 \mathrm{~nm}$ pore, called gel water, then, the amplitude of this $T_{2}$ component is simply a function of the amount of $\mathrm{C}-\mathrm{S}-\mathrm{H}$ in the paste and its position is
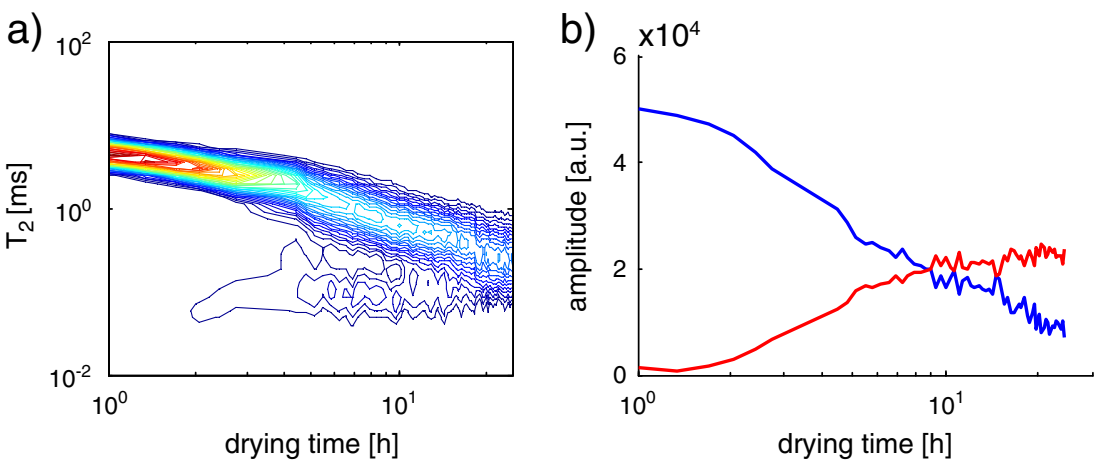

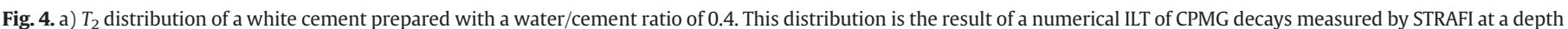

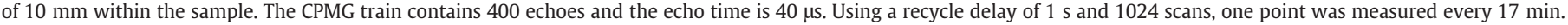

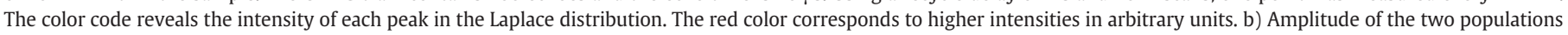

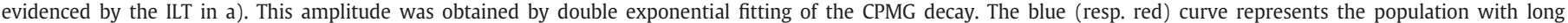
(resp. short) $T_{2}$. 

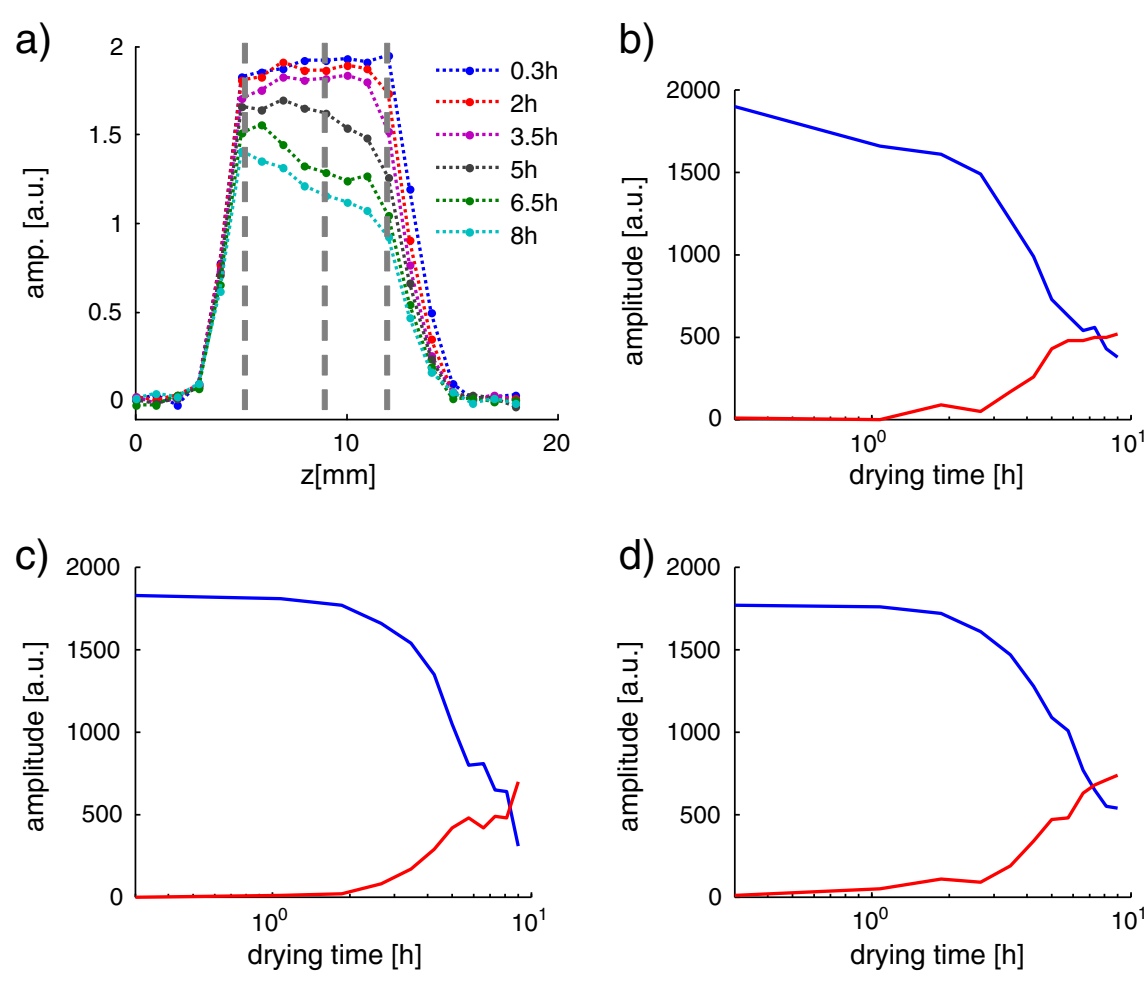

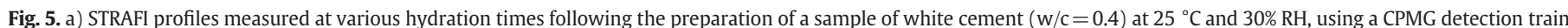

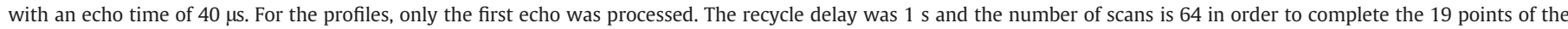

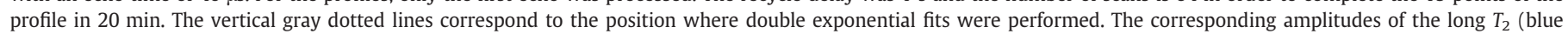
solid line) and short $T_{2}$ (red solid line) versus the hydration time are exhibited in plots (b) $\mathrm{z}=5 \mathrm{~mm}$, (c) $\mathrm{z}=9 \mathrm{~mm}$, and (d) $\mathrm{z}=12 \mathrm{~mm}$.

constant because the $S / V$ ratio of the gel is fixed. Despite the fact that this water is part of a pore, the size of the pore does not change dramatically in the $\mathrm{C}-\mathrm{S}-\mathrm{H}$. Moreover, this $\mathrm{T}_{2}$ component appeared late in the hydration process. Considering the experiment from Fig. 4 , it appears that the long $T_{2}$ value was decreasing continuously from $T_{2}=4 \mathrm{~ms}$ down to $T_{2}=400 \mu \mathrm{s}$. The evolution of the $T_{2}$ value with time (Fig. 4 ) and the evolution of the $T_{1}-T_{2}$ correlation maps (Fig. 6) yielded very similar results. These values are also the same as those found by Holly et al. [29] on a white cement hydrated with a w/s ratio of 0.42 . Besides getting access to the dynamics of the formation of the pore structure of white cement through the observation of the water distributions at a single position, STRAFI allows us to perform the same kind of study throughout the sample. Here, we showed for example (see Fig. 5) that the kinetics of the building of the pore structure of white cement was constant throughout the sample, even through evaporation induced a water gradient in the sample.

\subsection{The effect of temperature investigated with the Profile MOUSE}

Fig. 7 displays the NMR profiles obtained under $60 \%$ relative humidity at different hydration times and temperatures for a white cement paste. The sample was $22 \mathrm{~mm}$ thick and evaporation was allowed
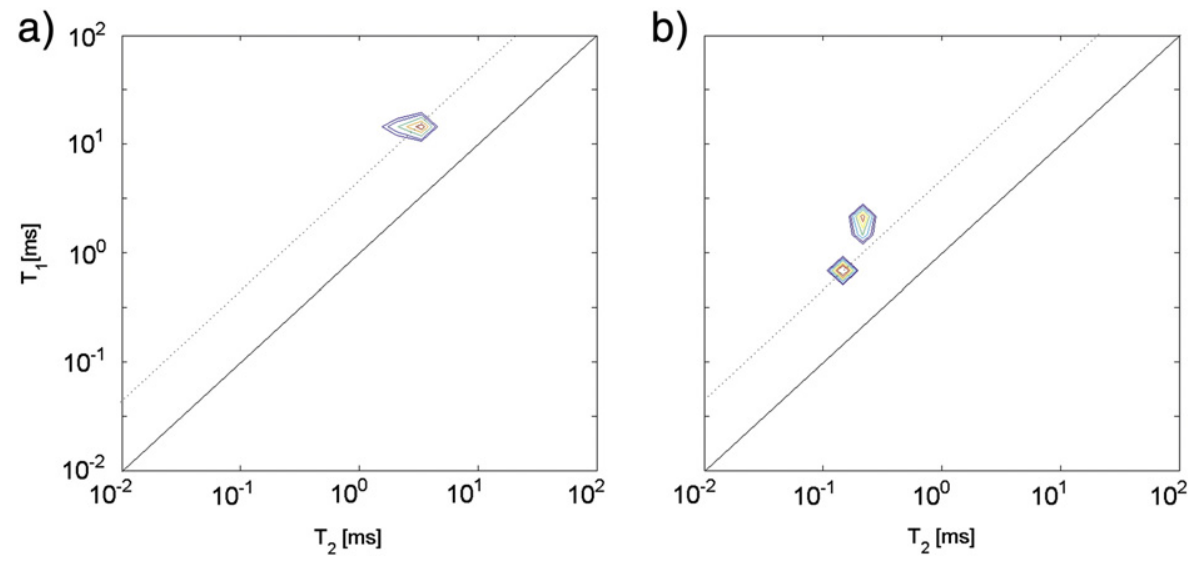

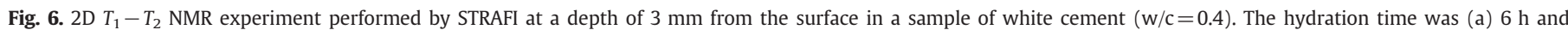

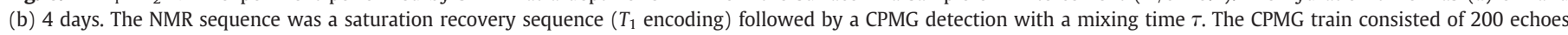

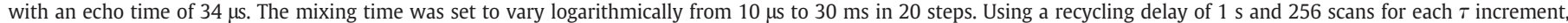
the time to measure the full 2D map was close to $1 \mathrm{~h}$. 
only on the upper face of the sample at the position $z=22 \mathrm{~mm}$. Two types of behavior could be distinguished depending on temperature. Below $25^{\circ} \mathrm{C}$, the profiles remained essentially flat except for a small region at the air interface of a few millimeters which is getting progressively dryer. The boundary of this region defines an evaporation front which progressed inward as drying proceeded. Above $25^{\circ} \mathrm{C}$, the behavior was the same during the first hours. However, behind the evaporation front, the profiles appeared progressively more tilted toward the air interface. To interpret theses data, it is important to recall that the Profile MOUSE setup is only sensitive to $T_{2}$ s larger than $500 \mu \mathrm{s}$. As shown in Fig. 3, this means that in a cement paste, the Profile MOUSE detects only the capillary water. From these considerations, we can conclude that below $20^{\circ} \mathrm{C}$, the amount of free water remained homogeneously distributed behind the evaporation front during hydration and drying except within a millimeter of the air interface where the capillary pores appeared totally desaturated. These observations were consistent with the results already observed by Bohris et al. [12] and are interpreted as follows: desiccation by evaporation was very effective next to the air interface where there is almost no capillary water. On the other hand, in the bulk of the sample, the amount of capillary water decreased due to drying and selfdesiccation but remains homogeneously distributed. The cement pore network efficiently redistributed the capillary water by capillary forces throughout the sample despite the unilateral evaporation. Behind the drying front, the drying mechanism is thus consistent with a funicular regime [28].

Above $25{ }^{\circ} \mathrm{C}$, the situation appeared significantly altered. Although a funicular drying mechanism can still be postulated at the beginning, after a certain time ( $4 \mathrm{~h}$ for instance at $35^{\circ} \mathrm{C}$ ), the amount of capillary water varied across the depth of the paste. At these temperatures, the pore network in the paste does not allow a timely capillary water transfer. We suggest that the drying mechanism changed to a pendular regime [28] around $25{ }^{\circ} \mathrm{C}$. Indeed, the effect of temperature can be either to induce a more efficient evaporation, or to speed up the hydration which prevents the capillary water transfer.

Fig. 8a and b exhibits the evolution of the amplitude and $T_{2}$ at various temperatures measured with the Profile MOUSE with respect to the hydration time of a white cement paste. The temperature affected drastically the kinetics of the setting of the cement paste. If one defines the setting as the inflection point of the curve amplitude versus drying time (Fig. 8a), we can see that it occurred at a drying time of about $20 \mathrm{~h}$ at $5{ }^{\circ} \mathrm{C}$ whereas it occurred at about $5 \mathrm{~h}$ at $35^{\circ} \mathrm{C}$. This result was consistent with the literature [30]. This evolution could also be seen in the change of $T_{2}$ with the drying time (Fig. 8b). Indeed, it exhibited a similar behavior. However, one can notice that the temperature affected not only the kinetics but also the values of $T_{2} \mathrm{~s}$. Fig. $8 \mathrm{~d}$ shows a linear dependence of $1 / T_{2}$ measured at the initial drying time as a function of temperature. This is interpreted as an effect of the temperature on the diffusion coefficient of water. Indeed, in a CPMG sequence performed in presence of a uniform magnetic field gradient $G$, the apparent $T_{2}$ is a function of the diffusion coefficient $\mathrm{tD}$ [31] which is a process activated with temperature (Eq. (3)).

When $T_{2}$ (Fig. 8b) is plotted as a function of the amplitude (Fig. 8a), one can see that all curves collapse to a single one (Fig. 8c) for all temperatures between $5{ }^{\circ} \mathrm{C}$ and $35^{\circ} \mathrm{C}$. The analysis of this master curve shows that it follows a power law with an exponent of 3 . This is interpreted in the following way: According to Eq. (1), the effective $T_{2}$ in a porous medium is a function of the ratio $S / V$ which is proportional to the pore size $r$ in spherical geometries. As far as the amplitude is concerned, it is proportional to the amount of spins in capillary pores. This amount depends of the pore volume and varies as $r^{3}$ in the case of
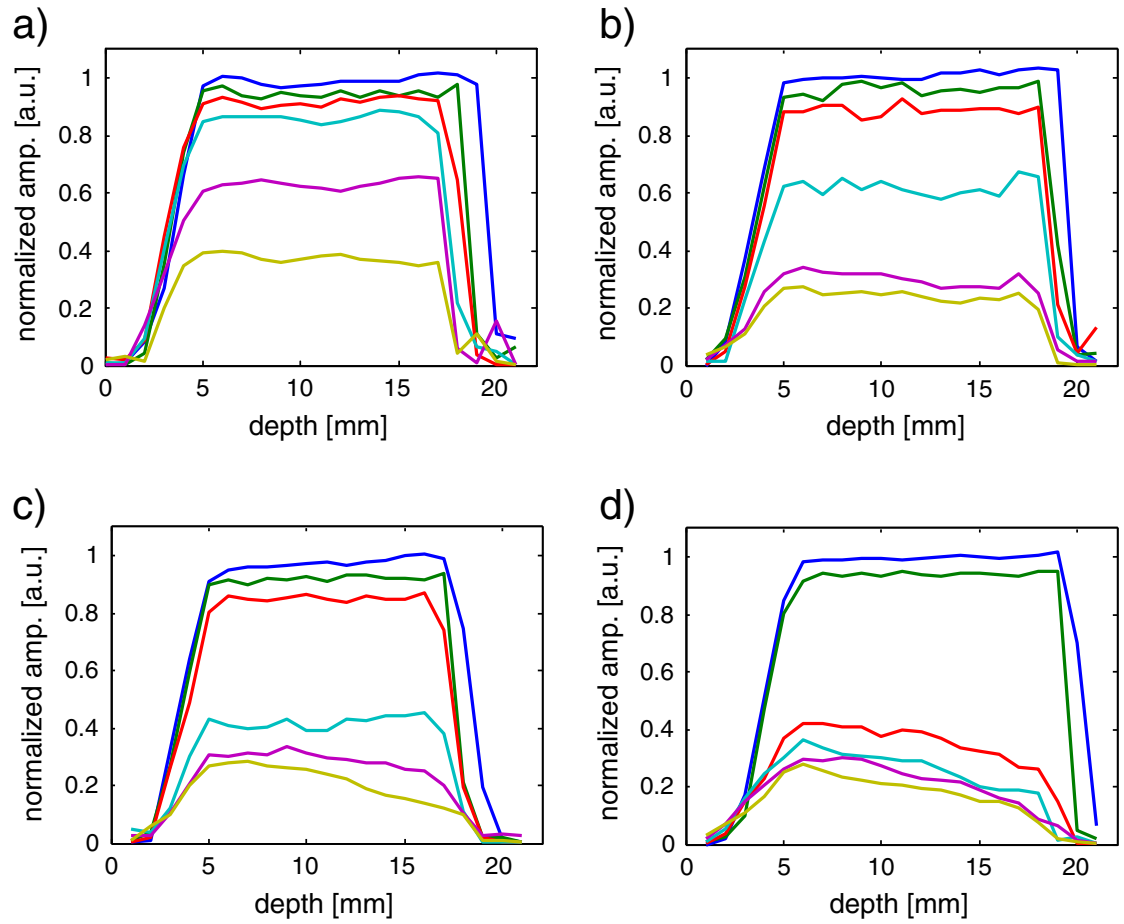

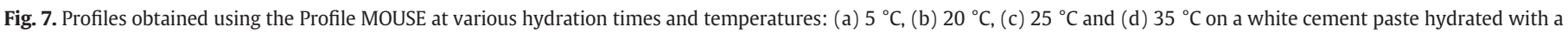

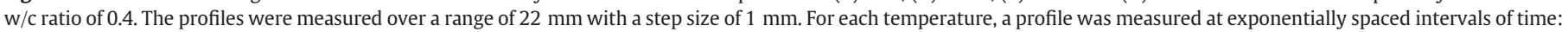

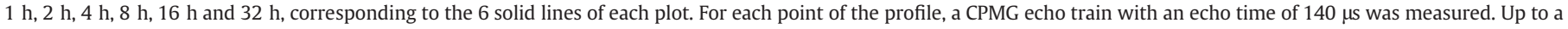

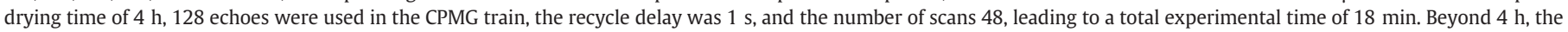

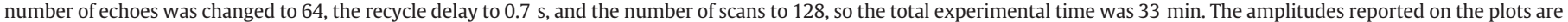
the amplitudes obtained from a single exponential fit of the CPMG decay. 
a)
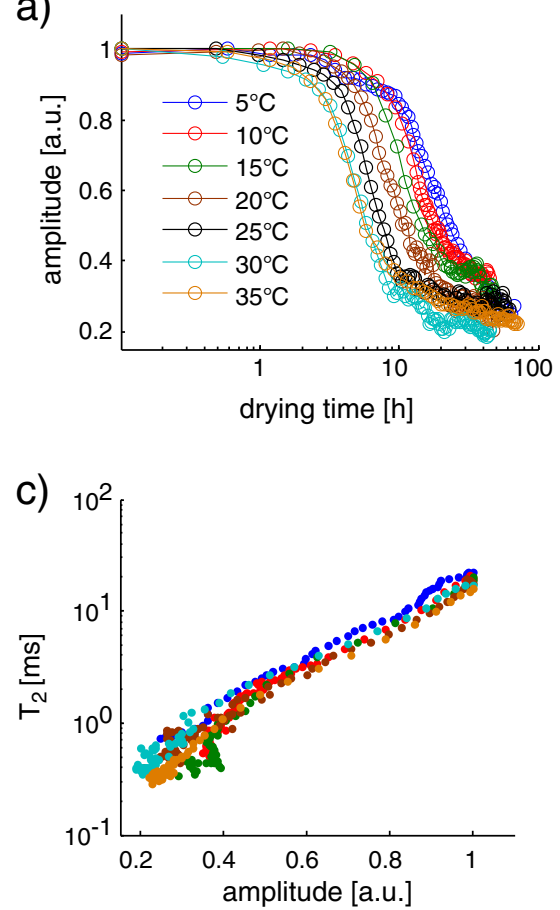

b)
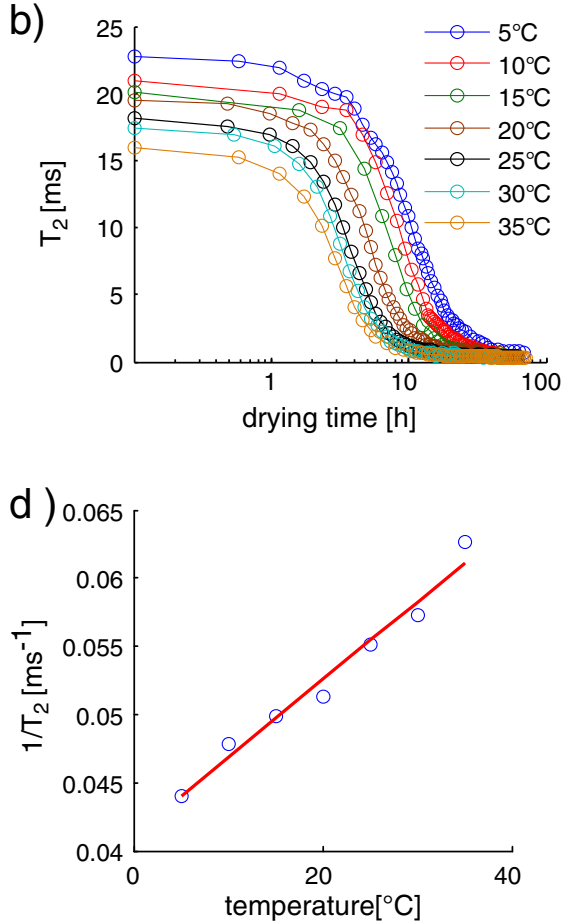

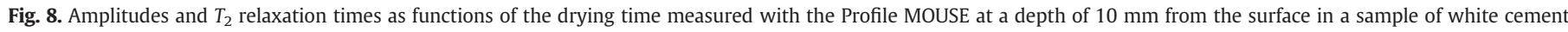

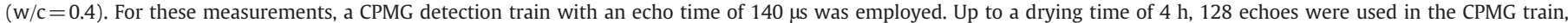

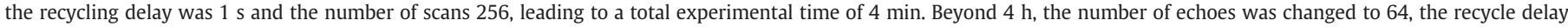

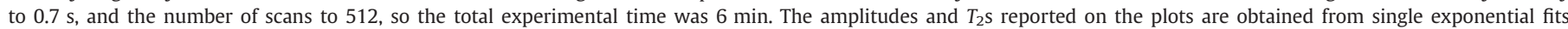

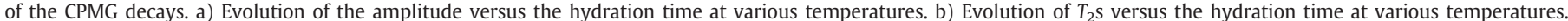
c) Correlation between amplitudes and $T_{2} \mathrm{~S}$ at various temperatures. d) Correlation between the relaxation rates measured at the initial drying time and temperature.

spherical pores. Thus, it sounds reasonable to find a power 3 law dependence of the apparent $T_{2}$ with the amplitude. This validates a posteriori the use of Eq. (1).

We showed that the Profile MOUSE is a very powerful tool which allows to observe simply the effect of drying and its consequence on the distribution of free water within the sample. In fact, it acts as a natural filter to exclusively observe the distribution of free water in white cement pastes. However, the disentanglement of drying and self-desiccation effects requires the use of the complementary STRAFI tool.

\subsection{Effect of relative humidity on the drying profiles}

At this stage, it can be argued that the NMR signal is not sensitive to the total amount of water. As we have seen before, $\mathrm{C}-\mathrm{H}$ and hydroxyl groups in the $\mathrm{C}-\mathrm{S}-\mathrm{H}$ have very short $T_{2}$ values (a few tens of microseconds typically). If a part of the Portlandite NMR signal is missed, the observed total amount of water should decrease with the hydrating time, even without any evaporation effect. In order to check this and obtain an evaluation of the missing signal, the paste was hydrated in a confined environment so that no water was lost (experiment at $100 \% \mathrm{RH}$ ). The result is displayed in Fig. 9. In this figure, one can see that the green curve slightly decreases with time. This decrease corresponds to a loss of $15 \%$ of the initial amplitude in $20 \mathrm{~h}$ of hydration. This effect is attributed to the conversion of free water into chemically combined water $(\mathrm{C}-\mathrm{H}$ and $\mathrm{C}-\mathrm{S}-\mathrm{H})$ whose $T_{2}$ is too short to be recorded.

To follow the influence of the humidity level on the desiccation of white cement paste, STRAFI profiles were acquired along the hydrating time at $22{ }^{\circ} \mathrm{C}$ and relative humidity of $20 \%$. For each position of the profile, the CPMG signal was fitted by a bi-exponential decay. The amplitude of both exponentials at two positions in the profile is displayed
Fig. 10. The effect of the drying is clearly evidenced. In the bulk (Fig. 10a), the decrease of free water (red squares) mainly corresponds as expected to a conversion of free water into gel water. The setting of the cement paste was observed at the hydration time $t=7 \mathrm{~h}$. Fig. 10b evidences a marked evaporation effect. Before a hydration time of $5 \mathrm{~h}$, the free water (red squares) strongly decreased because of evaporation. As a consequence, after the setting time, the weak amount of remaining free water was converted into gel water, and the drying rate was slowed down. As a noteworthy result, the behavior obtained for the bulk is the same for all positions in the paste. This is clearly

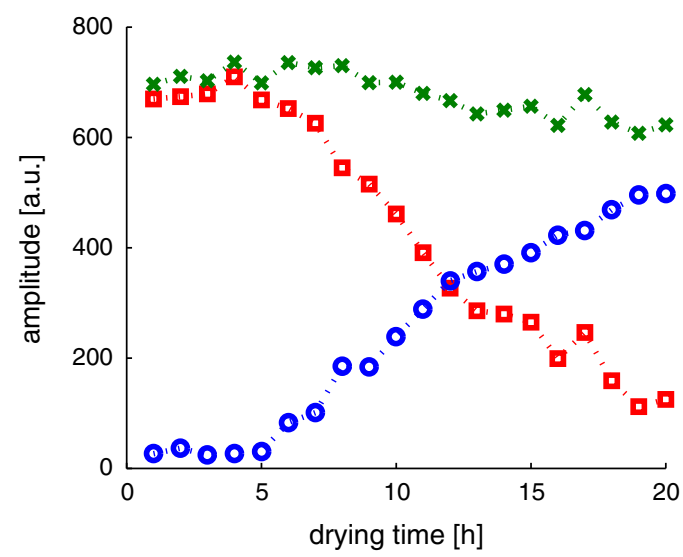

Fig. 9. Evolution of free (red squares) and "gel" (blue circles) water along the hydration time of a white cement paste $(\mathrm{w} / \mathrm{c}=0.4)$ at $22{ }^{\circ} \mathrm{C}$ and $100 \% \mathrm{RH}$. The observed slice was at a depth of $5 \mathrm{~mm}$ from the surface in the bulk. A CPMG detection train was performed with an echo time of $16 \mu$ s. The sum of both the free and the "gel" water is represented by the green line. 

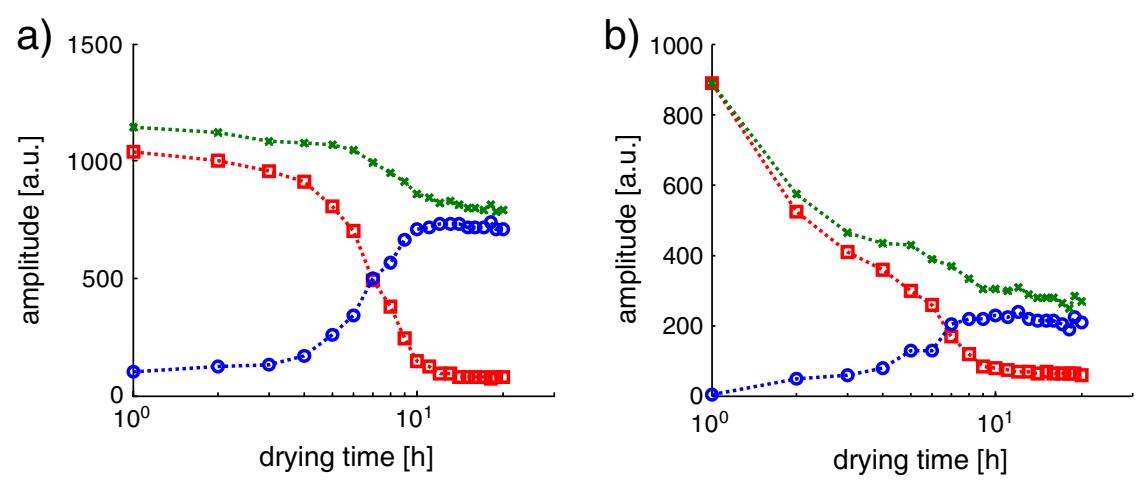

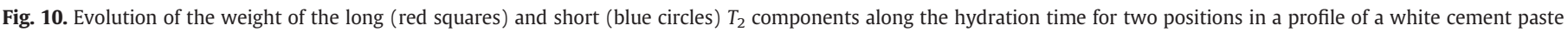

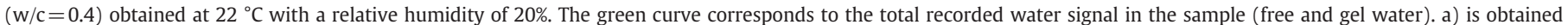
from a slice in the bulk of the paste, and b) is obtained at the surface (less than $1 \mathrm{~mm}$ from the surface).

consistent with the results obtained with the Profile MOUSE (Fig. 7): below $25^{\circ} \mathrm{C}$, the effect of the drying is effective up to a distance of $1 \mathrm{~mm}$ from the surface. For deeper positions in the paste, the water transport throughout the sample produced a homogeneous distribution of water in the paste. At this stage, it can be noticed that the total amount of free water in the bulk was slowly decreased. Indeed, it appears that before the setting of the paste, the bulk is slowly and homogeneously drying.

\section{Conclusion}

It is well known that the hydrating white cement paste develops a discrete porous network with a smallest pore diameter of $2.4 \mathrm{~nm}$. For the first time, the discrete behavior of a hydrating cement paste has been spatially resolved. The combination of two complementary techniques (the MOUSE and the STRAFI techniques) provided the opportunity to disentangle evaporation from hydration for different conditions of temperatures and relative humidity. While the STRAFI technique can detect the gel water as well as the capillary water, the MOUSE technique acts as a filter for the capillary water. Both techniques have been used to study the drying of a hydrating cement paste cylinder with one surface in contact with air.

The analysis of the spatially resolved $T_{2}$ distribution gave the following results: The $T_{2}$ of the water in the capillary pores progressively decreased from a few ms to $400 \mu$ s. The $T_{2}=150 \mu$ s of the gel water could be detected after $1 \mathrm{~h}$ and remained constant over time.

2D $T_{1}-T_{2}$ localized experiments have been successfully performed at the beginning of the hydration and after 4 days of hydration. The $T_{1}-T_{2}$ experiment allowed to validate the proton relaxation model in a STRAFI experiment. The 2D experiment gave the same $T_{2}$ distribution as in the simple STRAFI experiment. The discrete nature of the pore network was evidenced after 4 days.

Another conclusion could be drawn from the experiments at different temperatures. Below $25{ }^{\circ} \mathrm{C}$ the drying was homogeneous, the kinetics of structuring of the paste as well as the amount of water were the same whatever the position in the sample, except for a thin layer at the surface of the sample. The thickness of this layer varied with the drying time. It measured about $3 \mathrm{~mm}$ after $30 \mathrm{~h}$ of hydration. Above $25{ }^{\circ} \mathrm{C}$, the water profile was no longer homogeneous: On the contrary, the occurrence of a gradient of water content was consistent with a transition from a funical behavior toward a pendular behavior. At this temperature, the pore network was too dense to allow efficient capillary water transfer.

Two situations have been compared to evidence the effect of relative humidity. In the first case, the hydration occurred under a relative humidity of $100 \%$ and in the second case, the relative humidity was $20 \%$. A STRAFI experiment allowed to check that the loss of the water signal was due to the true drying process (capillary water evaporation) and not to the hydration (conversion of capillary water with "long" $T_{2}$ values into structural water of "short" $T_{2}$ values). The effect of the low relative humidity was to desaturate a layer at the surface of the drying sample. Elsewhere, the water distribution remained homogeneous.

\section{References}

[1] P. Coussot, Scaling approach of the convective drying of a porous medium, Eur. Phys. J. B. 15 (3) (2000) 557-566.

[2] P. Faure, P. Coussot, Drying of a model soil, Phys. Rev. E 82 (2010) 036303.

[3] H.F.W. Taylor, Cement Chemistry, Academic Press, 1990.

[4] F. Barberon, J.P. Korb, D. Petit, V. Morin, E. Bermejo, Probing the surface area of a cement-based material by nuclear magnetic relaxation dispersion, Phys. Rev. Lett. Chem. 90 (2003) 116103

[5] J.R. Zimmerman, W.E. Brittin, NMR studies in multiple phase systems: lifetime of a water molecule in an adsorbing phase on silica gel, J. Phys. Chem. 61 (1957) 1328-1333.

[6] W.P. Halperin, J.-Y. Jehng, Y.Q. Song, Application of spin-spin relaxation to measurement of surface area and pore size distribution in a hydrating cement paste, Magn. Reson. Imaging 12 (1994) 169-173.

[7] L. Venkataramanan, Y.Q. Song, M.D. Hürlimann, Solving Fredholm integrals of the first kind with tensor product structure in 2 and 2.5 dimensions, IEEE Trans. Signal Process. 50 (2002) 1017-1026.

[8] P.J. McDonald, J.P. Korb, J. Mitchell, L. Monteilhet, Surface relaxation and chemical exchange in hydrating cement pastes: a two-dimensional NMR relaxation study, Phys. Rev. E 72 (2005) 011409.

[9] P.J. McDonald, V. Rodin, A. Valori, Characterisation of intra- and inter-C-S-H gel pore water in white cement based on an analysis of NMR signal amplitudes as a function of water content, Cem. Concr. Res. 40 (2010) 1656-1663.

[10] J. Greener, H. Peemoeller, C. Choi, R. Holly, E.J. Reardon, C.M. Hansson, M.M. Pintar, Monitoring of hydration of white cement paste with proton NMR spinspin relaxation, J. Am. Ceram. Soc. 83 (2000) 623-627.

[11] T. Nunes, E.W. Randall, A.A. Samoilenko, P. Bodart, G. Feio, The hardening of Portland cement studied by ${ }^{1} \mathrm{H}$ NMR stray-field imaging, J. Phys. D: Appl. Phys. 29 (1996) 805-808.

[12] A.J. Bohris, U. Goerke, P.J. McDonald, M. Mulheron, B. Newling, B. Le Page, A broad line NMR and MRI study of water and water transport in Portland cement pastes, Magn. Reson. Imaging 16 (1998) 455-461.

[13] J. Boguszynska, M.C.A. Brown, P.J. McDonald, J. Mitchell, M. Mulheron, J. Tritt-Goc, D.A. Verganelakis, Magnetic resonance studies of cement based materials in inhomogeneous magnetic fields, Cem. Concr. Res. 35 (2005) 2033-2040.

[14] P.J. McDonald, Stray field magnetic resonance imaging, Prog. Nucl. Magn. Reson. Spectrosc. 30 (1997) 69-99.

[15] T. Nunes, Influence of grain size on the setting of Portland cement: a stray-field magnetic resonance imaging study, Mater. Sci. Forum 514-515 (2006) 1633-1637.

[16] P.J. McDonald, J. Mitchell, M. Mulheron, P.S. Aptaker, J.-P. Korb, L. Monteilhet, Two-dimensional correlation relaxometry studies of cement pastes performed using a new one-sided NMR magnet, Cem. Concr. Res. 37 (2007) 303-309.

[17] B. Blümich, J. Perlo, F. Casanova, Mobile single-sided NMR, Prog. Nucl. Magn. Reson. Spectrosc. 52 (2008) 197-269.

[18] M. Van Landeghem, B. Bresson, B. Blümich, J.-B. d'Espinose de Lacaillerie, Micrometer scale resolution of materials by stray-field magnetic resonance imaging, J. Magn. Reson. 211 (2011) 60-66.

[19] J. Perlo, F. Casanova, B. Blümich, Profiles with microscopic resolution by single-sided NMR, J. Magn. Reson. 176 (2005) 64-70.

[20] M.D. Hürlimann, D.D. Griffin, Spin dynamics of Carr-Purcell-Meiboom-Gill-like sequences in grossly inhomogeneous $B_{0}$ and $B_{1}$ fields and applications to NMR well logging, J. Magn. Reson. 143 (2000) 120-135. 
[21] E.W. Randall, A.A. Samoilenko, T. Nunes, Simultaneous ${ }^{1} \mathrm{H}$ and ${ }^{19} \mathrm{~F}$ stray-field imaging in solids and liquids, J. Magn. Reson. Ser. A 117 (1995) 317-319.

[22] In: F. Casanova, J. Perlo, B. Blümich (Eds.), Single-sided NMR, Springer, Berlin/Heidelberg, 2011.

[23] G. Goelman, M.G. Prammer, The CPMG pulse sequence in strong magnetic field gradients with application to oil-well logging, J. Magn. Reson. Ser. A 113 (1995) $11-18$.

[24] M.D. Hürlimann, L. Burcaw, Y.-Q. Song, Quantitative characterization of food products by two-dimensional $D T_{2}$ and $T_{1} T_{2}$ distribution functions in a static gradient, J. Colloid Interface Sci. 297 (2006) 303-311.

[25] P.J. McDonald, J. Mitchell, M. Mulheron, L. Monteilhet, J.-P. Korb, Two-dimensional correlation relaxation studies of cement pastes, Magn. Reson. Imaging 25 (2007) 470-473.

[26] J.L. Markley, W.J. Horsley, M.P. Klein, Spin-lattice relaxation measurements in slowly relaxing complex spectra, J. Chem. Phys. 55 (1971) 3604-3605.
[27] Y.-Q. Song, L. Venkataramanan, M.D. Hürlimann, M. Flaum, P. Frulla, C. Straley $T_{1}-T_{2}$ correlation spectra obtained using a fast two-dimensional Laplace inversion, J. Magn. Reson. 154 (2002) 261-268.

[28] C.J. Brinker, G.W. Scherer, Sol-Gel Science: The Physics and Chemistry of Sol-Gel Processing, Academic Press, London, 1990.

[29] R. Holly, E.J. Reardon, C.M. Hansson, H. Peemoeller, Proton spin-spin relaxation study of the effect of temperature on white cement hydration, J. Am. Ceram. Soc. 90 (2) (2007) 570-577.

[30] K.O. Kjellsen, R.J. Detwiler, Reaction kinetics of Portland cement mortars hydrated at different temperatures, Cem. Concr. Res. 22 (1992) 1101-1114.

[31] R. Kimmich, NMR: Tomography, Diffusometry, Relaxometry, Springer, Berlin/ Heidelberg, 1997. 